\title{
Design and Application of Railway Intelligent Big Data Information Network Platform
}

\author{
Guoxin Zhang ${ }^{1}$ *, Hongyu Lu' ${ }^{1}$, Hongjun Zhang ${ }^{2}$, Hongyan Zhang ${ }^{3}$ \\ ${ }^{1}$ Changchun Station, Shenyang Railway Bureau Group, China Railway, China \\ ${ }^{2}$ Department of Public Foreign Language Teaching and Research, Jilin University of Finance and Economics, \\ Jilin, China \\ ${ }^{3}$ Changchun Vocational and Technical School, China
}

Keywords: Information resource integration; Platform design; Development and application

\begin{abstract}
Integrating the independent information resources and management instructions of railway passenger station functional departments, intermediate stations, and production workshops into a unified system platform. The internal information of the platform is highly efficient and secure. Vertically, the infrastructure and application systems achieve unified and standardized management. Horizontally, Data sharing and system interconnection and interaction among various departments. This article describes the development background, design goals, network structure, overall design, and function implementation of the system platform, and then builds a unified big data network information platform to achieve smart office, resource sharing, scientific decision-making, and improve the railway intelligent transportation system. Level of decision support and control in various business areas.
\end{abstract}

\section{Introduction}

Nowadays, with the rapid development of artificial intelligence technology, computers and related technologies, countries have carried out research on transforming traditional railway transportation modes with new technologies [1]. The purpose is to improve the efficiency of railway transportation, enhance the safety of railway operations, improve service quality and reduce environmental pollution [2,3]. In recent years, major railway transportation countries such as Japan and Europe have actively invested in the research of the Railway Intelligent Transportation System (RITS) combined with rail transportation and Intelligent Transportation System (ITS). As China is actively carrying out research on the railway intelligent transportation system framework and related research, research on the railway big data platform and its architecture, integrate computer technology, network technology, data communication technology, integrate transportation production, daily office, management information system, and establish a unified The big data network informatization platform realizes intelligent office, resource sharing, scientific decision-making, and provides data support and guarantee for the safe production of railway transportation. It is of great significance to the reform and development of the country and the development of the railway transportation system itself.

\section{Status of China's Railway Intelligent Transportation System}

Railway is the main artery of China's national economy, and railway transportation occupies an extremely important position in the national economy. The railway transportation system directly affects the national economy, and it affects and counteracts market demand, which involves national security and stability. In order to strengthen the market competitiveness of China's railway transportation industry, improve management levels, improve marketing methods and service quality, China must vigorously carry out research on railway intelligent transportation systems. Although China has also developed comprehensive railway office systems (OMIS), railway transportation clearing systems, intelligent control of high-speed railway train operations, vehicle 
management information systems (CMIS), and financial management information systems (RFAMIS), these systems have been developed and connected independently. Poor interoperability and information can not be shared basically, resulting in a waste of resources. At present, the research on China's railway intelligent transportation system has certain basic technical conditions, but because it is still in its infancy, research and development lacks unified planning and deployment, especially for the overall structural system, organization, functions, standards, and logical structure And development methods need to do in-depth research and research.

This article takes the development and construction of Changchun Station integrated management information big data platform of Shenyang Bureau Group Company as the starting point, introduces the big data platform system in detail and analyzes and evaluates its architecture to provide a reference for the development of railway intelligent transportation systems.

\section{Technical Requirements and Hardware Environment for Big Data Platform R\&D}

According to the overall planning and implementation opinions of big data construction, integrate computer technology, network technology, and data communication technology, integrate transportation production, daily office, and management information systems, establish a unified big data network information platform, and realize intelligent office, resource sharing, Make scientific decisions to provide data support and guarantee for railway safety transportation production.

Big data platforms are mainly composed of clients, DMZ servers, application servers, and database servers. Application services and data services are isolated. App applications are implemented through DMZ servers through network technology and hardware limitations. The application server mainly handles request distribution and data packaging, and the database server mainly performs data processing.

The big data platform uses the B / S architecture design, which is characterized by no client installation, only a WEB browser, strong interactivity, no audit client, and only server upgrade. Send a request to the application server or App application to the DMZ server through a web browser, receive the request for distribution processing, and reasonably distribute the requested resource through load balancing technology, and then send it to the database server. The database server processes the data, returns it to the application server, and returns it to the client after the data packaging is complete. Realize data sharing with other external systems through technologies such as Web Service interface and Http interface, and database with the same name.

\section{Integrated Management Information Platform System Design}

\subsection{Platform construction software environment}

The comprehensive management information platform of Changchun Station was developed and constructed in the first half of 2014. After continuous development and improvement of three successive versions, as of the end of 2017, document circulation, technical regulations, safety management, equipment management, cost management, transportation operations, and cadre performance 15 sub-systems including employee performance, employee training, corporate culture, employee appeals, construction management, book management, public announcement and mobile APP, including 42 sub-modules including safety information analysis, safety early warning, cost budgeting, material management, etc. content.

\subsection{Frame structure}

The integrated management information platform architecture is a hierarchical structure that includes four layers: the network basic layer, the network basic service layer, the application support layer, and the information service layer (Figure 1). 


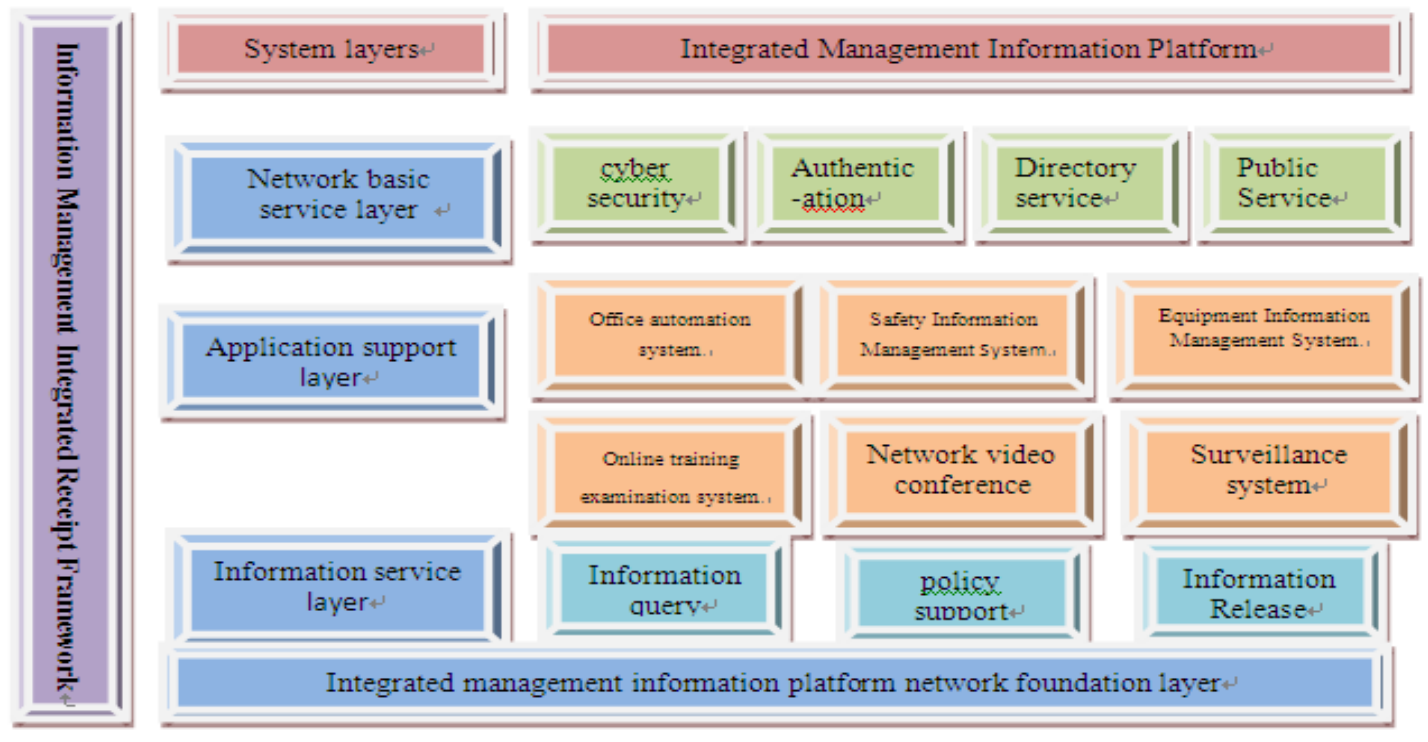

Figure 1 Platform architecture structure

\subsection{Logical structure design}

"Service-oriented" and "business modularization" are the overall design ideas for the system's logical structure. The logical structure of the system is divided into three parts: the presentation layer (UI user interface layer), application layer, and basic platform layer. The details are as follows pictured (Figure 2):

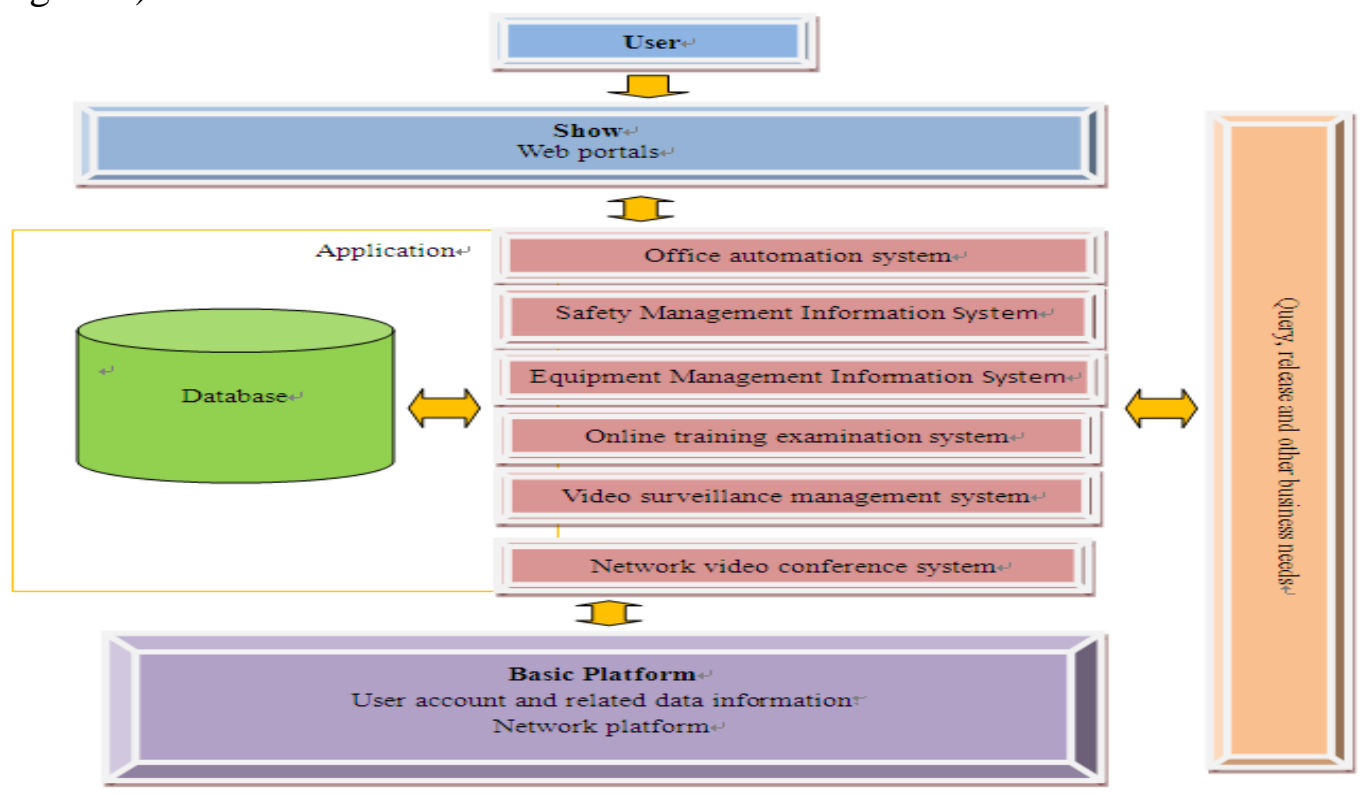

Figure 2 System platform logical structures

\subsection{Integrated Management Platform Application Subsystem}

\subsubsection{Safety management subsystem}

The safety management subsystem includes four modules: safety problem and hidden danger management, safety information analysis, safety warning and accident analysis electronic book. The security problem information comes from the real-time dynamic information found in the daily on-site inspection; the security information analysis includes security day, week, month analysis, and typical problem analysis, and all information realizes the automatic analysis function of big data; security warnings include security risk warnings, and workshop team failure Early warning and early warning of cadres 'performance; the accident analysis e-book includes accident information books, seedlings of driving accidents over the years, statistics on safety days, and 
employees' rewards and punishments.

\subsubsection{Technical regulation subsystem}

The Technical Regulation Subsystem contains four modules: regulatory text, emergency disposal, technical information, and staff post operation specifications. It implements real-time dynamic updating of technical information, electronic management of technical information, and processization of emergency disposal.

\subsubsection{Equipment management subsystem}

The equipment management subsystem includes four modules: travelling equipment, passenger equipment, special equipment and other equipment. All equipment is implemented with two-dimensional code management. Each equipment has an identification two-dimensional code. On-site faulty equipment can dynamically manage and control the equipment problem library by scanning the two-dimensional code. At the same time, the fault is discovered by Unicom maintenance unit and repaired in time. Account electronic resume management, equipment from procurement, operation, maintenance, maintenance to maintenance at a glance.

\subsubsection{Cost management subsystem}

The cost management subsystem includes four modules: budget submission, material application, financial system and material management. The budget submission starts from the submission of the budget by the on-site application department, and undergoes dynamic electronic approval of each link from budget approval, budget verification, budget execution to on-site verification, and each budget is implemented in a two-way dual-track system; material applications range from application materials to material approval, material procurement, materials The verification, material inquiry, and usage verification links also implement a two-way dual-track system; the financial system mainly reflects the relevant institutional measures of the unit's property; the material management includes the details of the unified procurement of the material management platform and the place of use.

\subsubsection{Performance assessment subsystem}

The performance appraisal subsystem includes cadre performance and staff performance. The cadre performance includes on-site quantitative inspection, monthly problem detection, daily job performance, integrated assessment, and monthly evaluation of the workshop. Staff performance includes daily assessment, Business examination, workload salary and performance summary.

\subsubsection{Staff training subsystem}

The employee training subsystem includes four modules: training plan, comprehensive analysis, online education, and comprehensive testing. The daily employee training is dynamically connected to on-site issues, and targeted training is developed. Through the establishment of online courses such as quality courses and risk point interpretation, the training is expanded. Channels, through the background of automatic data analysis of employee technical files, use error analysis to reinforce training, so as to achieve multi-channel, multi-mode training purposes, and played a good effect in daily practical training.

\subsubsection{Construction management subsystem}

The construction management subsystem includes construction plans, regular construction meetings, construction drills, and coordinated unit management to achieve the purpose of electronic signing of construction and dynamic real-time drills before construction operations.

\subsubsection{Basic management subsystem}

The basic management subsystem includes modules such as basic information, labour books, comprehensive books, meeting records, etc. It focuses on the electronic management of various basic books and realizes paperless office. 


\subsubsection{Mobile APP functions}

It mainly realizes the real-time positioning of cadres' on-site inspections, real-time entry of problems, and real-time viewing of content such as job performance assessment, dynamic information, and equipment management of the web version of employees.

\section{Prospect of Railway Intelligent Transportation System}

With the rapid development of computer and network communication technology, especially the large-scale popularization of the Internet, the research of artificial intelligence is in full swing and is developing in a wider field. The research and development around intelligent application systems also usher in a new period of vigorous development. The railway intelligent transportation system is the development goal of the railway transportation system. Intelligent railways and intelligent trains are another sign of progress based on the current digital railways and digital trains.

At present, neural network can be used to automatically generate speed mode control curves, and hierarchical multivariable fuzzy control can be used to achieve safe, efficient, energy-saving, punctual and comfortable train operation requirements; evolutionary computation and neural networks can be used to realize driving and shunting operations The automation and intelligence of dispatch and command; the use of neural networks and fuzzy expert systems can establish intelligent transportation decision support systems to assist leadership decisions. As a large-scale intelligent information processing system, the railway intelligent transportation system must have "intelligence" in its self-organizing behaviour. Its intelligent embodiment should include three aspects: the intelligence of subsystems (agents) and the collective decision-making ability of each subsystem and the evolution of intelligence [4,5].

\section{Conclusion}

The intelligence requirements of various information systems in the railway transportation system have not yet emerged. The life cycle of these information systems has just begun, and their ability to cope with complex problems has not been tested. Therefore, making full use of the results of the existing intelligent system theory, and improving the level of decision support and control of various business areas in the railway intelligent transportation system will be the main direction for the development of railway intelligent transportation systems.

\section{References}

[1] Lu Huapu. Green Transportation: The Direction of Sustainable Development of Urban Transportation in China. Comprehensive Transportation. 2011(02).

[2] Gao Jinjun, Lu Li. Policy design and practice progress of low-carbon transportation, Transportation Enterprise Management 2012-05-15.

[3] Harremoes, P., Topsoe, F.: Maximum entropy fundamentals. Entropy 3, 191-226 (2001).

[4] Yang, H., Lin, L., Yu, and Z.: A class of fuzzy multiple attributes TOPSIS decision making based on exponential type fuzzy numbers. Compute. Eng. App. 48(34), 120-124 (2012).

[5] Li, C-Y., Xu, M.-Q.: The importance analysis of equipment based on the improved TOPSIS.J. Vib. Shock 28(6), 19-27 (2009). 